\title{
Prediction for multiaxial fatigue strength with small defects
}

\author{
Keiji Yanase ${ }^{1,2^{*}}$ and Masahiro Endo ${ }^{1,2}$ \\ ${ }^{1}$ Department of Mechanical Engineering, Fukuoka University, Fukuoka City, Fukuoka, Japan \\ ${ }^{2}$ Institute of Materials Science and Engineering, Fukuoka University, Fukuoka City, Fukuoka, Japan
}

\begin{abstract}
As an extension of previous studies, this paper further examine the applicability and characteristics of the predictive method, in particular, for the case of proportional loading. The predictive method is based on the $\sqrt{\text { area }}$-parameter model and Endo's biaxial fatigue failure criterion. The present study manifests that the critical plane is normal to the direction of maximum principal stress, and the fatigue strength is dictated by both the maximum and minimum principal stresses.
\end{abstract}

\section{Introduction}

In recent decades, the ability to assess the effect of small defects, inclusions and inhomogeneities on the uniaxial fatigue strength has been improved rapidly [1]. However, the structural components in service are often subjected to multiaxial fatigue loading (e.g., combined axial and torsional loading in a shaft). Therefore, it is of practical merit to propose a predictive model that can connect the fatigue strength under multiaxial loading with that under uniaxial loading.

According the previous studies by the authors, the multiaxial fatigue strength in the presence of small surface defect can be predicted by the following equation [2-4]:

$$
\max \left\{\Sigma_{\mathrm{w}}(\theta)\right\}=\sigma_{\mathrm{w}}=\frac{1.43(H V+120)}{\left(\sqrt{\text { area }}_{\max }\right)^{1 / 6}}
$$

where, in the case of proportional loading:

$$
\sum_{\mathrm{w}}(\theta)=\left\|\left(\cos ^{2} \theta+\kappa \sin ^{2} \theta\right) \cdot \sigma_{0}+(1-\kappa) \sin ^{2} \theta \cdot \tau_{0}\right\|
$$

In Eq. (2), $\sigma_{0}$ and $\tau_{0}$ signify the stress amplitudes by axial and torsional loadings, respectively, and a parameter $\kappa(=-0.18)$ accounts for the effect of stress biaxility. Figure 1 illustrates the procedures to predict the fatigue strength. In essence, Eq. (1) can be classified as the critical plane approach.

\section{Results and Discussions}

Based on a necessary condition to find the fatigue strength (Fig. 1), the critical angle, $\theta$ cri, is calculated as:

\footnotetext{
*Corresponding author: kyanase@fukuoka-u.ac.jp
} 


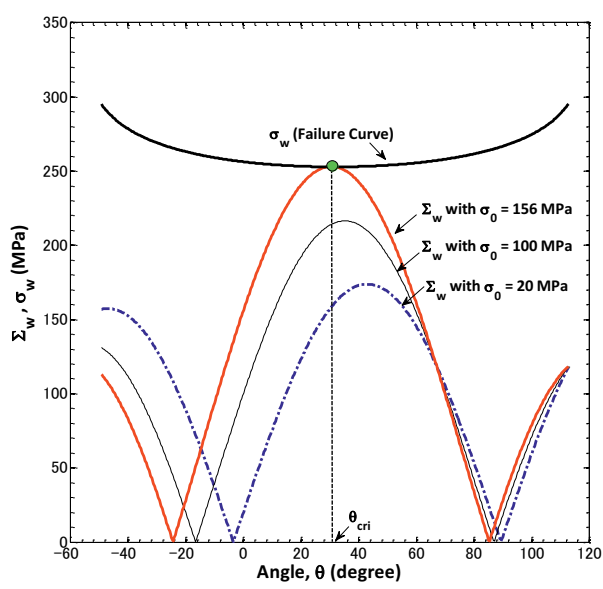

(a) Prediction for normal stress amplitude, $\sigma_{0}$

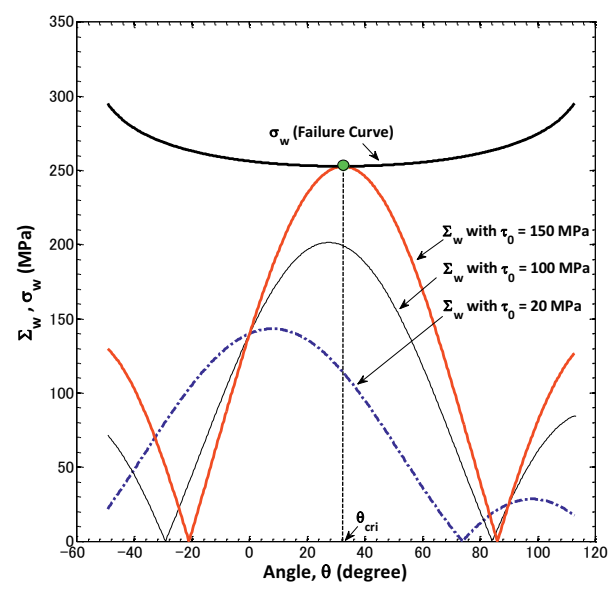

(b) Prediction for shear stress amplitude, $\tau_{0}$

Figure 1. Schematic illustration to find the fatigue strength.

$$
\left.\frac{\partial \sum_{\mathrm{w}}(\theta)}{\partial \theta}\right|_{\theta=\theta_{c r i}}=0 \quad \rightarrow \quad \theta_{c r i}=\frac{1}{2} \tan ^{-1}\left(\frac{2 \tau_{0}}{\sigma_{0}}\right)
$$

In practice, $\theta_{c r i}$ in Eq. (3) represents the principal stress direction. Further, by substituting Eq. (3) into Eq. (1), the following equation can be obtained:

$$
(1+\kappa) \frac{\sigma_{0}}{\sigma_{\mathrm{w}}}-\kappa\left(\frac{\sigma_{0}}{\sigma_{\mathrm{w}}}\right)^{2}+(1-\kappa)^{2}\left(\frac{\tau_{0}}{\sigma_{\mathrm{w}}}\right)^{2}=1
$$

By using the experimental data, the predictive capability of Eq. (4) is examined in Fig. 2. As shown, a reasonable accuracy is observed.

Moreover, by using the maximum and minimum principal stresses, $\sigma_{\mathrm{n}}$ (normal to critical plane) and $\sigma_{\mathrm{p}}$ (parallel to critical plane), Eq. (4) leads to the following equation:

$$
\sigma_{\mathrm{nw}}=\left(1+\kappa \frac{\sigma_{\mathrm{p}}}{\sigma_{\mathrm{n}}}\right)^{-1} \times \frac{1.43(H V+120)}{\left(\sqrt{\text { area }}_{\max }\right)^{1 / 6}}
$$

Eq. (5) coincides with a phenomenological equation proposed by Yanase and Endo [5]. As manifested by Eqs. (3) and (5), the orientation of critical plane is independent of $\kappa$ but the fatigue strength is affected by $\kappa$.

\section{References}

1. Y. Murakami, Metal fatigue: Effects of small defects and nonmetallic inclusions, Elsevier (2002).

2. M. Endo, I. Ishimoto, Int. J. Fatigue 28, pp. 592-597 (2006).

3. K. Yanase, ASTM Materials Performance and Characterization 2(1), DOI: 10.1520/MPC20130013 (2013).

4. M. Endo, K. Yanase, Theoretical and Applied Fracture Mech. 69, pp.34-43 (2014).

5. K. Yanase, M. Endo, Engng. Fract. Mech., (submitted).

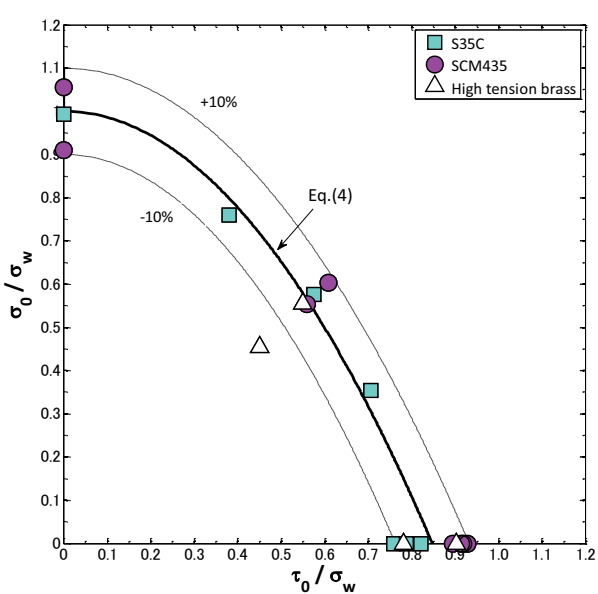

Figure 2. Predictions with Eq. (4). 